country of origin was not recorded at death. It was constructed based on numbers of foreign-born in specific agegroups at each census employing a novel actuarial method. For example, if $\mathrm{p}$ is the mortality rate for the $\mathrm{fb}$ age 30 per year in the interval 1947-1954 then $\mathrm{p}$ is estimated from the data by 1- (number all fb age 35-45 in 1954/number all $\mathrm{fb}$ age $25-35$ in 1947$)^{1 / 7}$. $p$ for other age-groups is calculated similarly. For all age-groups $\mathrm{p}$ will underestimate the mortality rate.

Results The Australian population grew exponentially between 1861 and 1986, reaching 15,602,150 million; its demographic composition was $64.7 \%$ foreign born initially and of predominantly British and Irish origin. Later twentieth century immigration was from mainly Southern European and Asian countries and FB accounted for a smaller proportion (22.4\%) of the population by 1986. A strong period effect across all age groups and both genders was observed for CSD mortality. Rates of CSD rose consistently, particularly from the 1940 s onwards, peaked in the 1960 s and then began to decline sharply in the 1980s. Both male and female foreign-born showed similar all-cause mortality patterns at age midpoints from 30 through to 60 years of age.

Conclusion Our findings support both a commonly experienced period effect for CSD mortality but suggest also that early origins of foreign-born may contribute to mortality patterns.

\section{P75 CONTEXTUAL INFLUENCES ON CHRONIC DISEASE: A MULTI-LEVEL ANALYSIS IN THE TWIN CITY OF RAMALLAH AND AL BIREH IN THE OCCUPIED PALESTINIAN TERRITORY}

Ahmad Alkhatib*, Jonathan Olsen, Richard Mitchell. MRC/CSO Social and Public Health Sciences Unit, University of Glasgow, Glasgow, UK

\subsection{6/jech-2021-SSMabstracts. 163}

Background The features of the urban environment can support human health as well as harm it, but less is known about the context of middle eastern countries. The association between green space and the political classification of the urban environment and the risk of chronic illness was investigated in a novel setting, the twin city of Ramallah and Albireh in the occupied Palestinian territory.

Methods We used multi-level regression analysis to link the 2017 census data with contextual characteristics from Geographic Information System data. The outcome variable is the presence or absence of chronic illness. The contextual explanatory variables are: the proportion of three types of green space in the areas of residence (mixed trees, crop trees and open space with little or no vegetation); the locality of residence (Ramallah, Albireh and Refugee camps); and political land classification (Area A, B, and C). Generalised multi-level analysis was conducted in $\mathrm{R}$ using 'lme4' package, with individuals at level one $(n=54693)$ and areas of residence at level two $(n=228)$, adjusting for individual demographic and socioeconomic characteristics.

Results Just over $13 \%$ of the chronic illness risk variation was attributed to the area of residence. On the political dimension and while accounting for the individual-level factors, only living in the context of a refugee camp had a significant positive association (OR 1.91 CI [1.17-3.09]). However, the association between refugee camps and chronic illness rendered non-significant when green space variables were entered into the model. In the final model, only the proportions of 'mixed' tress in the residential areas had a significant inverse association with the risk of chronic illness (OR $0.96 \mathrm{CI}$ [0.95-0.97]), all else being equal.

Conclusion Within the confines of a cross-sectional, observational study, the findings shed light on the possible role of the urban environment context as a determinant of health, irrespective of individual-level factors. Our ability to differentiate between several types of green space was important, as findings demonstrated that not all types are associated with reduced risk of chronic illness. Our results from a middle eastern setting add to the existing evidence, largely in Western countries, that trees in urban settings are important and beneficial to human health. Also, to reduce inequalities in health researchers and policymakers should give more attention to the disadvantaged contexts of refugee camps.

\section{P76 INCIDENCE, OUTCOMES AND CHARACTERISTICS OF OUT-OF-HOSPITAL CARDIAC ARRESTS IN PATIENTS WITH PSYCHIATRIC ILLNESS: A SYSTEMATIC REVIEW}

${ }^{1}$ Raied Alotaibi* ${ }^{1}$ 'Laura Bijman, ${ }^{1}$ Nynke Halbesma, ${ }^{2}$ Gareth Clegg, ${ }^{1}$ Caroline Jackson. 'Usher Institute, University of Edinburgh, Edinburgh, UK; ${ }^{2}$ Resuscitation Research Group, University of Edinburgh, Edinburgh, UK

\subsection{6/jech-2021-SSMabstracts. 164}

Background People with a history of psychiatric illness have a lower life expectancy than people without psychiatric illness, largely due to an excess burden of cardiovascular disease (CVD). Despite the well-established association between psychiatric illnesses and risk of CVD in general, little is known about psychiatric illness relates to the incidence and outcomes of out-of-hospital cardiac arrest (OHCA) specifically. We therefore aimed to conduct a systematic review of the literature to critically appraise and summarise the existing evidence on incidence, outcomes and characteristics of OHCA in patients with psychiatric illness.

Methods We searched Embase, Medline, PsycINFO and Web of Science from the first publication within each database to 16th December 2020 using a detailed electronic search strategy containing a wide range of terms for psychiatric illness and OHCA. We included observational studies that reported on the characteristics of patients with OHCA or OHCA incidence or survival by psychiatric illness status (or that reported on patients with psychiatric illness without including a comparison group). Two authors independently screened the search results, assessed risk of bias in relevant studies and extracted data. We registered the protocol of this review with PROSPERO (CRD42021229545).

Results Our search retrieved 10,610 potentially eligible studies, of which nine met our inclusion criteria. Of these, eight were retrospective cohort studies and one was a case-control study comprising 5,906 OHCA patients with history of psychiatric illness across five countries. Three studies included patients with depression only, whilst six studies varied in their definition of psychiatric illness. Eight studies reported on psychiatric illness status with respect to OHCA incidence and only one study assessed OHCA outcome. Most studies found that psychiatric illness was associated with an increased risk of OHCA. Among patients with an OHCA, those with 
psychiatric illness were more likely to be female, younger and have non-shockable rhythms compared to patients without psychiatric illness. No study reported on the distribution of comparison groups by socioeconomic status, which is known to be linked to both psychiatric illness and poorer OHCA survival. The only study on OHCA survival reported lower odds of 30-day survival in patients with versus without psychiatric illness.

Conclusion This review highlights the paucity of studies reporting on psychiatric illness in relation to OHCA incidence and outcome. History of psychiatric illness may be a risk factor for OHCA incidence and poorer outcome, but further studies are needed in this clearly under-researched, yet very important, area.

\section{P77 MODELLING THE SPREAD OF BEHAVIOURAL RISK FACTORS FOR CARDIOVASCULAR DISEASE IN A UK COMMUNITY USING AN AGENT-BASED MODEL}

${ }^{1}$ Marion Dugue*, ${ }^{1}$ Nathan Griffiths, ${ }^{1}$ James Archbold, ${ }^{2}$ Oyinlola Oyebode. ${ }^{1}$ Department of Computer Sciences, University of Warwick, Coventry, UK; ${ }^{2}$ Warwick Medical School, University of Warwick, Coventry, UK

\subsection{6/jech-2021-SSMabstracts. 165}

Background Tobacco use, unhealthy diet, physical inactivity, and harmful use of alcohol are key behavioural risk factors for non-communicable diseases, including cardiovascular disease. Individuals' behaviours and attitudes are affected by their social environment, which can lead to the spreading of a behavioural risk factor through social connections. Understanding the spread of behavioural risk factors through social networks may allow us to identify targets amenable to intervention to reduce risk factor prevalence and ultimately death and suffering from non-communicable disease. Agentbased models are under-utilised as a tool to give insight into the epidemiology of non-communicable diseases.

Methods We propose an agent-based model of the spread of four behavioural risk factors through social networks (defined as friendships and household relationships), based on the linear threshold cascade model. We created a synthetic community of agents each assigned a unique ID, an age (in three agegroups: $18-34,35-64$ and $65+$ years), sex, and a number of connections in the relevant communities (friendships and household contacts), as well as a level ([0-2], based on increasing risk of CVD) for each of the behaviours modelled. Parameters were evidence-informed estimates, based on epidemiological data published in the literature and expert opinion. Where possible nationally representative data from the UK or England were used to estimate parameters. We used the Python package NetworkX to create the network.

Results The method was applied to find the population risk of cardiovascular disease over 10 years for a population size of 10,000 . Our model estimated rates of cardiovascular disease from 1.3 events per 1000 person years in 1839 year old women to 83.1 events per 1000 person years in $65+$ year old men which are consistently smaller than rates from observed data. We found that the model was most sensitive to the estimates of influence of spouse behaviour on agent behaviour. It was also sensitive to the average number of household contacts, particularly for the 18-34 age-group.

Conclusion Future work is needed to address our model's limitations which include that the model only considers two communities for each agent: its household and its friendships. Other communities such as workplaces and neighbourhoods could be integrated in the future. Future improvements will also be to add ethnicities and deprivation level to agents. Interactions between multiple behaviours are also relevant here. Our simplistic model assumes no interactions between concepts but integrating this in future versions will be necessary to model closer to reality.

\section{P78 USE OF MATHEMATICAL MODELLING FOR POLICY MAKING: EXAMPLE OF CHRONIC KIDNEY DISEASE IN CHILE}

${ }^{1}$ Magdalena Walbaum*, 'Shaun Scholes, ${ }^{2}$ Ruben Rojas, ${ }^{1}$ Jennifer Mindell, ${ }^{3}$ Elena Pizzo. ${ }^{1}$ Research Department of Epidemiology and Public Health, University College London, London, UK; ${ }^{2}$ School of Health and Related Research, University of Sheffield, Sheffield, UK; ${ }^{3}$ Applied Health Research, University College London, London, UK

\subsection{6/jech-2021-SSMabstracts. 166}

Background Chronic Kidney Disease (CKD) is a leading public health problem, with substantial burden for individuals, healthcare systems and society. Predictive tools to simulate the disease in the future could help inform decision makers of potential impacts of new treatments, for effective policy making. The aim of this study was to compare the impact of introducing pre-dialysis treatment in Chile with the standard treatment using a multistate model to estimate the future cases and costs of CKD.

Methods A dynamic stock and flow model was used to simulate CKD progression in the Chilean population aged $\geq 40$ years, to the year 2030, from the Chilean public healthcare system perspective. The model included six states replicating progression of CKD, which was assumed in 1-year cycles and was categorised as slow or fast progression. We compared the current treatment for CKD Stages $3 \mathrm{a}$ to End-Stage Kidney Disease (ESKD) covered in Chile with a scenario that introduced pre-dialysis treatment for CKD Stages 4 and 5. Only direct treatment costs were considered. The model was calibrated based on international evidence; 95\% credibility intervals were calculated with probabilistic sensitivity analysis. We conducted a financial analysis to calculate the annual cash flow and net present value with $3 \%$ and $6 \%$ discount rates. Results By the year 2030, there is an expected increase in cases of CKD Stages 3a to ESKD, ceteris paribus, from $452,198$ (95\% CI $332,760-571,636)$ in 2020 to 558,271 $(483,997-632,545)$ individuals. Direct costs of CKD stages $3 \mathrm{a}$ to ESKD would rise from $£ 256.2 \mathrm{M}$ GBP $(148.8-363.6)$ in 2020 to $£ 516.6 \mathrm{M}(359.5-693.6)$ in 2030 . The introduction of pre-dialysis treatment for CKD Stages 4 and 5 would reduce the proportion of fast progressors from the $30 \%$ assumed in the baseline scenario to $20 \%$. This intervention is estimated to decrease the number of individuals worsening to stages 5 and ESKD, and reduce the total costs of CKD healthcare by $£ 82.6 \mathrm{M}$ in 2030 to $£ 434.0 \mathrm{M}(277.1-591.0)$. The financial analysis showed a net present value of $-£ 139.8 \mathrm{M}$ and $-£$ $117.0 \mathrm{M}$ with $3 \%$ and $6 \%$ discounts, respectively.

Conclusion Predictive models are a useful tool for decisionmaking. The inclusion of pre-dialysis treatment for CKD Stages 4 and 5 would generate savings for the healthcare system due to the reduction in progression of CKD to ESKD. These results were presented to policy makers (Health, then Treasury) in Chile, to consider including pre-dialysis treatment for Stages 4 and 5 in the funded CKD healthcare in Chile. 